\title{
A Modally Adaptive Control for Multi-Contact Cyclic Motions in Compliantly Actuated Robotic Systems
}

\author{
Dominic Lakatos, Martin Görner, Florian Petit, Alexander Dietrich, and Alin Albu-Schäffer
}

\begin{abstract}
Compliant actuators in robotic systems improve robustness against rigid impacts and increase the performance and efficiency of periodic motions such as hitting, jumping and running. However, in the case of rigid impacts, as they can occur during hitting or running, the system behavior is changed compared to free motions which turns the control into a challenging task. We introduce a controller that excites periodic motions along the direction of an intrinsic mechanical oscillation mode. The controller requires no model knowledge and adapts to a modal excitation by means of measurement of the states. We experimentally show that the controller is able to stabilize a hitting motion on the variable stiffness robot DLR Hand Arm System. Further, we demonstrate by simulation that the approach applies for legged robotic systems with compliantly actuated joints. The controlled system can approach different modes of motion such as jumping, hopping and running, and thereby, it is able to handle the repeated occurrence of robot-ground contacts.
\end{abstract}

\section{InTRODUCTION}

Intrinsic elasticities in biological and robotic multi-body systems enable the execution of highly dynamic and complex motions, such as hitting and throwing, or walking and running. The compliant actuator behavior improves the mechanism robustness during rigid contacts with the environment and increases performance and energy efficiency. However, the generation of effective and efficient motions is not trivial. In this work we aim at robustly controlling periodic motions for robotic systems with variable stiffness actuation (VSA) [1], [2], [3]. The idea is to exploit the natural dynamics of these robotic systems and to control them such that they are able to robustly handle contacts, in a similar way as their biological archetype. However, due to environmental contacts and the variable stiffness mechanism, the open-loop system dynamics are strongly nonlinear. Therefore, the basic assumption is that the considered systems intrinsically feature internal nonlinear oscillation modes, which correspond to motion patterns mentioned above. The goal of this paper is to find an appropriate control strategy, which firstly identifies and secondly excites one of these oscillation modes such that the repeated occurrence of contacts can even be used to preload the springs.

In our previous work [4] we analyzed the oscillatory behavior of VSA robotic arms. We showed that the existence of quasi-independent oscillation modes strongly depends on the availability of sufficient damping in parallel to the springs, which ensures the decay of oscillations excited in the other modes. From observations of humans controlling a compliantly actuated system by force/visual feedback, we derived a simple bang-bang controller, which is able to excite and sustain periodic motions for such systems [4]. The controller is triggered by a generalized force and

The authors are with the Institute of Robotics and Mechatronics, German Aerospace Center (DLR), D-82234 Oberpfaffenhofen, Germany. Contact: dominic.lakatos@dlr.de switches the equilibrium position of the spring in a selected joint. Thereby, energy gets injected into the system and oscillations are excited. Since the controller requires almost no system knowledge, it performs very robustly and is predestined for systems with model uncertainties, including robot-environment contacts.

The goal of this work is to extend the ideas initiated in [4] for systems and tasks including robot-environment contacts, with a focus on legged robotic systems. Our conclusions for legged systems go along with different experimentally supported hypotheses of biologists [5] and related work on compliant quadrupeds [6]. Full and Koditschek [5] hypothesize that fast locomotion is dominated by the mainly feedforward controlled mechanical system and its interaction with the environment. Following their arguments, the high-dimensional, nonlinear system dynamics anchored in a complex animal collapse to simple template dynamics like the spring loaded inverted pendulum (SLIP). This requires that coupled, nonlinear, neural and mechanical oscillators synchronize in phase and excite coordinated periodic motions. These hypotheses are further supported by the work of Ijspeert [7] and Buchli et al. [6]. In [7] multiple neural oscillators are coupled to a compliant segmented mechanics model of a salamander. By proper parametrization, the model shows coordinated traveling and standing waves along the body that result in swimming and walking motions, respectively. The work in [6] shows that adaptive frequency oscillators are able to find, to adapt to and to enforce intrinsic, mechanical modes of locomotion of a quadruped with rigidly actuated hip and passively compliant knee joints.

The main contribution of this paper is the extension of the single-input single-output controller presented in [4] to the multi-input multi-output case. This is achieved by an adaptive part which converges to a coordinate transformation of the dominant oscillation mode of the plant. The coordinate transformation is then used to modally distribute the energy input over the joints and thereby increases the effectiveness of the limit cycle excitation. The modally adaptive control strategy is validated for robotic systems in the presence of contacts. In an initial experimental test with a multi-joint VSA robotic arm, we demonstrate that the controller is able to stabilize a cyclic hammering motion. Then, we apply the control approach to the simulation of a legged system with compliant actuation in the joints and show that jumping and running motions can be excited easily. In contrast to [6] or [8], [9], which address rigid actuators or full rigid body motions, respectively, we aim at exciting the mechanical intrinsic oscillation modes. Hereby, a main difference is that we consider systems with compliant actuators for all joints. We propose (building up on [4]) a completely new approach of adaptive feedback controlled modal excitation. It is a fundamentally different concept from the basically 
open loop intrinsic pattern generators, even in their version with adaptive oscillation frequency [6], trying to mimic the fast adaptive nature of biological motion generation. The introduced method offers the capability to distribute the energy input over the joints and to achieve the directed excitation of a specific oscillation mode, using an adaptive feedback control, which approaches an excitation in the intrinsic frequency of the system and task. Thereby, the adaptive part accounts for the distribution of the excitation amplitudes (similar to the eigenvectors in linear oscillation theory) such that they fit to and properly excite an intrinsic oscillation mode of the system.

The paper is structured as follows: In Section II we introduce the control approach. Then, we experimentally demonstrate that the controller can handle contacts for a hitting motion (Section III). In Section IV we perform a simulation study with a legged system. A brief conclusion is given in Section $\mathrm{V}$

\section{Controller Design}

First, we revisit the bang-bang control introduced in [4]. Then, we propose an extension to multi-joint robots, using an adaptive law, which converges to the coordinate transformation corresponding to a dominant oscillation mode of the plant.

\section{A. Bang-bang control for a single compliantly actuated joint}

Consider the model of a single compliantly actuated joint

$$
\begin{aligned}
I \ddot{q}+d \dot{q} & =\tau, \\
b \ddot{\theta}+\tau & =\tau_{m},
\end{aligned}
$$

where $q, \theta \in \mathbb{R}$ are link and motor coordinates, respectively, $\tau_{m}$ is the control input, and

$$
\tau=\psi(\theta-q)
$$

is the joint torque. The motor inertia $b$ acts over a nonlinear spring $\psi(\theta-q)$ on the link inertia $I$. Additionally, viscous damping $d \dot{q}$ acts on the link. Considering the control $\tau_{m}=$ $-k_{\mathrm{D}} \dot{\theta}-k_{\mathrm{P}} \theta+k_{\mathrm{P}} \theta_{d}$ (where $k_{\mathrm{P}}$ and $k_{\mathrm{D}}$ are positive controller gains), the singular perturbation assumption [10]:

$$
\epsilon\left(b \ddot{\theta}+\tau+k_{\mathrm{D}} \dot{\theta}\right)=\theta_{d}-\theta,
$$

with $\epsilon=1 / k_{\mathrm{P}}$ and $\epsilon \rightarrow 0$, reduces (1)-3) to

$$
I \ddot{q}+d \dot{q}+\psi(q-\theta)=0,
$$

where $\theta$ is the new control input.

Our goal to excite and stabilize periodic motions in the link coordinate $q$ can be achieved by the discontinuous control law

$$
\theta\left(q, \theta_{-}\right)=\left\{\begin{array}{cc}
\operatorname{sign}\left(\tau\left(q, \theta_{-}\right)\right) \hat{\theta} & \text { if }\left|\tau\left(q, \theta_{-}\right)\right|>\epsilon_{\tau} \\
0 & \text { otherwise }
\end{array}\right.
$$

where $\epsilon_{\tau}>0$ is a threshold, $\hat{\theta}$ a constant switching amplitude and $\theta_{-}$the state of $\theta$ before the switching. It is worth mentioning that, if the joint torque $\tau$ or equivalently the deflection $(\theta-q)$ can be measured, the controller (6) will not require any model knowledge.

\section{B. Modeling VSA robotic systems}

Variable stiffness actuated robots can be represented by Euler-Lagrange equations [11], satisfying

$$
\frac{\mathrm{d}}{\mathrm{d} t}\left(\frac{\partial L(\boldsymbol{x}, \dot{\boldsymbol{x}})}{\partial \dot{\boldsymbol{x}}}\right)-\frac{\partial L(\boldsymbol{x}, \dot{\boldsymbol{x}})}{\partial \boldsymbol{x}}=\tau_{\mathrm{gen}}-\boldsymbol{d}(\boldsymbol{x}, \dot{\boldsymbol{x}}),
$$

where the Lagrangian $L(\boldsymbol{x}, \dot{\boldsymbol{x}})=T(\boldsymbol{x}, \dot{\boldsymbol{x}})-U(\boldsymbol{x})$ is the difference of the kinetic energy $T(x, \dot{x})$ and potential energy $U(\boldsymbol{x})$. The potential energy $U(\boldsymbol{x})=U_{g}(\boldsymbol{x})+U_{\psi}(\boldsymbol{x})$ is the sum of a gravity and elastic potential, respectively. The position states $\boldsymbol{x}=\left(\boldsymbol{\theta}^{T}, \boldsymbol{q}^{T}\right)^{T} \in \mathbb{R}^{m+n}$ can be divided into motor positions $\boldsymbol{\theta} \in \mathbb{R}^{m}$ and link positions $\boldsymbol{q} \in \mathbb{R}^{n}$. The vector of generalized forces $\tau_{\text {gen }}=\left(\boldsymbol{u}^{T}, \boldsymbol{\tau}_{\text {ext }}^{T}\right)^{T}$ is composed of the control $\boldsymbol{u} \in \mathbb{R}^{m}$ and the external torques $\tau_{\text {ext }} \in \mathbb{R}^{n}$. Only the motor states $\left(\boldsymbol{\theta}^{T}, \dot{\boldsymbol{\theta}}^{T}\right)^{T}$ are directly actuated via the control input $\boldsymbol{u}$. Moreover, $\boldsymbol{d}(\boldsymbol{x}, \dot{\boldsymbol{x}}) \in \mathbb{R}^{m+n}$ represents a damping force, where $\dot{\boldsymbol{x}}^{T} \boldsymbol{d}(\boldsymbol{x}, \dot{\boldsymbol{x}}) \geq 0$ holds.

This model represents a large class of compliantly actuated robots and highlights the generality of the control approach proposed in the remainder of the paper.

In the following experimental and simulative evaluation, we consider serial elastic actuation. Thereby, the elastic potential $U_{\psi}=U_{\psi}\left(\boldsymbol{x}, \boldsymbol{\theta}_{\psi}\right)$ depends on parameters $\boldsymbol{\theta}_{\psi} \in \mathbb{R}^{m}$ to adjust the stiffness characteristics. In this work we deal with constant stiffness preset (although nonlinear), i.e. $\dot{\boldsymbol{\theta}}_{\psi}=\mathbf{0}$.

\section{Adapting the modal coordinate transformation}

Our goal is to control periodic motions in the link position coordinates $\boldsymbol{q} \in \mathbb{R}^{n}$ using the bang-bang control (6) as presented above for the single joint case. Since the controller accounts only for scalar quantities, we seek a transformation such that the motion $\boldsymbol{q}(t)$ can be represented by a single coordinate, for instance, $y_{1}(t)$. The basic idea is sketched in Fig. 1 and will be explained in a context of differential geometry as follows. Consider the time series of joint positions $\boldsymbol{q}(t)$ representing the motion of the multi-joint robot, where $\boldsymbol{q} \in Q \subset \mathbb{R}^{n}$ are coordinates of a manifold $Q$. Assume that we can represent the trajectory $\boldsymbol{q}(t)$ on a lower dimensional manifold $Y$, with coordinates $\boldsymbol{y} \in Y \subset \mathbb{R}^{p \leq n}$. (In particular, for the bang-bang control it is required that $p=1$.) Assume further that the mapping

$$
\boldsymbol{y}=\boldsymbol{F}(\boldsymbol{q}, \boldsymbol{W})
$$

can be parameterized by constant weights $\boldsymbol{W}$ and the inverse mapping

$$
q=G(y, W)
$$

exists. Then, similar to what was done in [12], we can define an error function

$$
S=\|\boldsymbol{q}(t)-(\boldsymbol{G} \circ \boldsymbol{F})(\boldsymbol{q}(t), \boldsymbol{W})\|^{2} .
$$

For a perfect reconstruction mapping, the sum of error functions evaluated at each point of the trajectory $\boldsymbol{q}(t)$ must be identically zero. In general, this leads to a nonlinear optimization problem, where the matrix of weights $\hat{W}$ represents the optimal solution.

To clarify the meaning of the reduction mapping (8), let us review the linear, second order system

$$
\boldsymbol{M} \ddot{\boldsymbol{q}}+\boldsymbol{K} \boldsymbol{q}=\mathbf{0},
$$




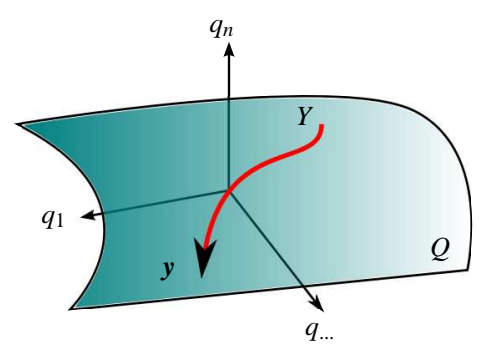

Fig. 1. The idea of the modal transformation

where $\boldsymbol{M}, \boldsymbol{K} \in \mathbb{R}^{n \times n}$ are constant, symmetric, and positive definite matrices. With eigenvectors $\hat{\boldsymbol{w}}_{i} \in \mathbb{R}^{n}$ of the matrix丩 $\boldsymbol{A}:=\boldsymbol{K}^{-1 / 2} \boldsymbol{M} \boldsymbol{K}^{-1 / 2}$ (where $\hat{\boldsymbol{w}}_{i}$ are normalized such that $\hat{\boldsymbol{w}}_{i}^{T} \hat{\boldsymbol{w}}_{j}=\delta_{i j}$ and $\delta_{i j}$ is the Kronecker delta), the motion of the system (11) can be expressed as

$$
\boldsymbol{q}(t)=\hat{\boldsymbol{w}}_{1} y_{1}(t)+\hat{\boldsymbol{w}}_{2} y_{2}(t)+\ldots+\hat{\boldsymbol{w}}_{n} y_{n}(t),
$$

where $y_{i}(t)=\hat{a}_{i} \sin \left(\omega_{i} t-\phi_{i}\right)$ are time modulations of the eigenmodes corresponding to motions along $\hat{w}_{i}$. Hereby, $\hat{a}_{i}$ are amplitudes, $\omega_{i}$ eigenfrequencies and $\phi_{i}$ phase angles. From (12) it can be seen that

$$
\boldsymbol{q}=\sum_{i} \boldsymbol{w}_{i} y_{i}
$$

where $\boldsymbol{w}_{i} \in \mathbb{R}^{n}$ are parameters of the mappings (13) and $\boldsymbol{q} \in \mathbb{R}^{n}$ and $y_{i} \in \mathbb{R}$ represent the instantaneous values of the trajectory $\boldsymbol{q}(t)$ and $y_{i}(t)$, respectively. Due to orthogonality of $\boldsymbol{w}_{i}$, the modal reduction mapping has the form

$$
y_{i}=\boldsymbol{w}_{i}^{T} \boldsymbol{q} .
$$

Remark 1: Equation (13) is the representation of the linear differential equation (11) as a superposition of independent modal oscillations. If only one mode is excited, the motion can be exactly represented by only a single coordinate $y_{i} \in \mathbb{R}$ and $S$ in 10 becomes zero.

Remark 2: In the general case of nonlinear mappings (8) respectively (9), differential geometry provides clear rules how to transform contravariant vectors (velocities) and covariant vectors (forces) between the manifolds $Q$ and $Y$ [13]. For instance, velocity vectors transform with the Jacobian, i.e. $\dot{\boldsymbol{q}}=(\partial \boldsymbol{G}(\boldsymbol{y}) / \partial \boldsymbol{y}) \dot{\boldsymbol{y}}$ and force co-vectors with the transposed Jacobian, i.e. $\boldsymbol{\tau}_{y}=(\partial \boldsymbol{G}(\boldsymbol{y}) / \partial \boldsymbol{y})^{T} \tau_{q}$. In the case of linear, orthogonal transformations (13) respectively (14), the transformation matrix is equal to the Jacobian, e.g. $\partial \boldsymbol{G}(\boldsymbol{y}) / \partial \boldsymbol{y} \equiv \boldsymbol{W}$. Moreover, $\boldsymbol{W}^{-1} \equiv \boldsymbol{W}^{T}$. It is worth mentioning that in this paper we will always apply the clear rules of differential geometry also to the nonlinear case.

With the above considerations in mind, let us now derive an adaptive law for the linear system (11), which converges to the parameters $\boldsymbol{w}_{i}$ of the mappings (13), (14) under the assumption of unknown $\boldsymbol{K}$ and $\boldsymbol{M}$. Assume therefore that we measure a new value of the actual joint position $\boldsymbol{q}(k)$ at each

\footnotetext{
${ }^{1}$ The matrix $A:=\boldsymbol{K}^{-1 / 2} \boldsymbol{M} \boldsymbol{K}^{-1 / 2}$ results from the transformation $\boldsymbol{q}=$ $\boldsymbol{K}^{-1 / 2} z$, i.e. $\ddot{z}+\boldsymbol{A} z=\mathbf{0}$, where $\boldsymbol{A}$ is still symmetric and positive definite. The eigenvectors of $\boldsymbol{A}$ are related to the generalized eigenvectors of $\boldsymbol{K}, \boldsymbol{M}$ [14, chap. 4.5].
}

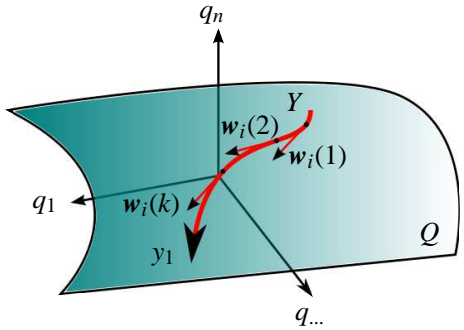

Fig. 2. Local approximation of the modal transformation

discrete time instance $k$. Consider further the error function

$$
S=\frac{1}{4}\left\|\boldsymbol{q}(k)-\sum_{i} \boldsymbol{w}_{i} \boldsymbol{w}_{i}^{T} \boldsymbol{q}(k)\right\|^{2},
$$

which represents the squared distance between the input $\boldsymbol{q}(k)$ and the auto associative mapping $\boldsymbol{w}_{i} \boldsymbol{w}_{i}^{T} \boldsymbol{q}(k)^{2}$ at time instance $k$. Then, the gradient descent rule

$$
\tilde{\boldsymbol{w}}_{i}(k)=\tilde{\boldsymbol{w}}_{i}(k-1)-\gamma \frac{\partial S\left(\boldsymbol{q}(k), \boldsymbol{w}_{i}(k-1)\right)}{\partial \boldsymbol{w}_{i}},
$$

where $\gamma>0$ determines the convergence rate and

$$
\frac{\partial S\left(\boldsymbol{q}(k), \boldsymbol{w}_{i}(k-1)\right)}{\partial \boldsymbol{w}_{i}}=-y_{i}(k)\left(\boldsymbol{q}(k)-y_{i}(k) \boldsymbol{w}_{i}(k-1)\right),
$$

minimizes the error function (15) recursively and provides a new guess $\tilde{\boldsymbol{w}}_{i} \in \mathbb{R}^{n}$ at each time instance $k$. (Note that $\left.y_{i}(k)=\boldsymbol{w}_{i}(k-1)^{T} \boldsymbol{q}(k)\right)$. Since the algorithm (16), (17) does not ensure orthogonality of the weights $\tilde{\boldsymbol{w}}_{i}$, we incorporate the Gram-Schmidt orthogonalization (see, e.g. [14, chap. $0.6]$ ) as implicit constraints:

$$
\begin{gathered}
\overline{\boldsymbol{w}}_{i}(k)=\tilde{\boldsymbol{w}}_{i}(k)-\sum_{j<i} \boldsymbol{w}_{j}(k-1)^{T} \tilde{\boldsymbol{w}}_{i}(k) \boldsymbol{w}_{j}(k-1), \\
\boldsymbol{w}_{i}(k)=\left(\overline{\boldsymbol{w}}_{i}(k)^{T} \overline{\boldsymbol{w}}_{i}(k)\right)^{-1 / 2} \overline{\boldsymbol{w}}_{i}(k) .
\end{gathered}
$$

Thereby, the step from $\tilde{\boldsymbol{w}}_{i}$ to $\overline{\boldsymbol{w}}_{i} \in \mathbb{R}^{n}$ performs the orthogonalization and the latter step normalizes $\overline{\boldsymbol{w}}_{i}$ such that $\boldsymbol{w}_{i}^{T} \boldsymbol{w}_{j}=\delta_{i j}$. Assuming that $\gamma \ll 1$ and neglecting terms of order $O\left(\gamma^{2}\right)$, we obtain the learning rule

$$
\begin{aligned}
\boldsymbol{w}_{i}(k)= & \boldsymbol{w}_{i}(k-1)+\gamma y_{i}(k)\left[\boldsymbol{q}(k)-y_{i}(k) \boldsymbol{w}_{i}(k-1)\right. \\
& \left.-2 \sum_{j<i} y_{j}(k) \boldsymbol{w}_{j}(k-1)\right],
\end{aligned}
$$

proposed by [15].

Remark 3: The $p$ dominant eigenvectors $\hat{\boldsymbol{w}}_{1}, \ldots, \hat{\boldsymbol{w}}_{p}$ of the data covariance matrix $\boldsymbol{C}=E\left(\boldsymbol{Q} \boldsymbol{Q}^{T}\right)$, where $\boldsymbol{Q}=$ $[\boldsymbol{q}(1), \boldsymbol{q}(\cdots), \boldsymbol{q}(k)] \in \mathbb{R}^{n \times k}$, represent asymptotically stable fixed-points of the difference equations (18). The proof therefore is given in [16], [17].

Remark 4: For the linear system (11), the eigenvectors $\hat{\boldsymbol{w}}_{1}, \ldots, \hat{\boldsymbol{w}}_{p}$ of the data covariance matrix $\boldsymbol{C}$ are related to the oscillation modes, i.e. $\hat{\boldsymbol{w}}_{i}$ are eigenvectors of the matrix $\boldsymbol{A}$ which are represented in the particular motion. In the

\footnotetext{
${ }^{2}$ The auto associative mapping $\boldsymbol{w}_{i} \boldsymbol{w}_{i}^{T} \boldsymbol{q}(k)$ is the composition of the reduction mapping 14 and the inverse mapping 13.

${ }^{3}$ The operator $E(\cdot)$ denotes the expectation value of the argument.
} 
presence of damping, the eigenvectors of the matrix $\boldsymbol{C}$ approximate the eigenvectors of the resonant modes. These properties are proven, respectively discussed in [18].

As can be seen in (7), the link side dynamics of a multijoint robotic system are nonlinear. Therefore, the notion of eigenmodes as in the case of linear systems might be replaced by so-called nonlinear normal modes [19]. For the present approach, we assume that the dominant mode is synchronous in amplitudes (i.e. the oscillations of the joints are in phase) such that the motion along this mode can be represented by a single curvy-linear coordinate. Therefore, when the algorithm described by (18) converges sufficiently fast, the weight vector $\boldsymbol{w}_{i}(k)$ approximates the instantaneous linearization of the nonlinear normal mode. This is sketched in Fig. 2 Finally, it is worth mentioning that the order of the weight vectors $\boldsymbol{w}_{i}(k)$ depend on the motion $\boldsymbol{q}(k)$, which is the only "information source" of the adaptation algorithm. The first weight vector $\boldsymbol{w}_{1}$ corresponds to the most dominant principal component of the trajectory $\boldsymbol{q}(k)$, i.e. to the eigenvalue of the matrix $\boldsymbol{C}$ with the largest magnitude.

\section{Bang-bang control in a modal direction}

Let us now assume that we want to excite periodic motions around the center $\boldsymbol{\theta}_{0} \in \mathbb{R}^{m}$. Consider therefore the PD control

$$
\boldsymbol{u}=-\boldsymbol{K}_{D} \dot{\boldsymbol{\theta}}-\boldsymbol{K}_{P}\left(\boldsymbol{\theta}-\boldsymbol{\theta}_{d}\right),
$$

where $\boldsymbol{K}_{D}, \boldsymbol{K}_{P} \in \mathbb{R}^{m \times m}$ are symmetric and positive definite controller gain matrices, and $\boldsymbol{\theta}_{d} \in \mathbb{R}^{m}$ is a new control input, i.e. the desired motor position, for the system (7). Then, using $\boldsymbol{w}_{1} \in \mathbb{R}^{m}$, provided by the adaptation algorithm (18), we can compute the bang-bang control in the direction of the first mode:

$$
\Delta \theta_{z}\left(\tau_{z}\right)=\left\{\begin{array}{cc}
\operatorname{sign}\left(\tau_{z}\right) \hat{\theta}_{z} & \text { if }\left|\tau_{z}\right|>\epsilon_{\tau_{z}} \\
0 & \text { otherwise }
\end{array} .\right.
$$

Herein,

$$
\tau_{z}=\boldsymbol{w}_{1}^{T}\left(\frac{\partial U_{\psi}(\boldsymbol{\theta}, \boldsymbol{q})}{\partial \boldsymbol{\theta}}-\left.\frac{\partial U_{\psi}(\boldsymbol{\theta}, \boldsymbol{q})}{\partial \boldsymbol{\theta}}\right|_{\boldsymbol{\theta}=\boldsymbol{\theta}_{0}}\right) \in \mathbb{R}
$$

is the generalized force acting in the direction of the first mode, $\epsilon_{\tau_{z}} \in \mathbb{R}_{>0}$ the corresponding threshold and $\hat{\theta}_{z} \in \mathbb{R}$ the modal switching amplitude. Finally, the control input $\boldsymbol{\theta}_{d}$ in (19) has the form:

$$
\boldsymbol{\theta}_{d}=\boldsymbol{\theta}_{0}+\Delta \boldsymbol{\theta}
$$

Thereby, we interpret $\Delta \theta_{z}\left(\tau_{z}\right) \in \mathbb{R}$ as a tangent vector of the modal manifold such that it transforms with the Jacobian $\boldsymbol{w}_{1}$ of the inverse reduction mapping (13), i.e. $\Delta \boldsymbol{\theta}=\boldsymbol{w}_{1} \Delta \theta_{z}\left(\tau_{z}\right)$.

Remark 5: As discussed in Section [II-C $\boldsymbol{w}_{1}$ converges to a local approximation of the nonlinear mode. Therefore, the control (18) and (19)-(22) slightly excites the other, "undesired" modes. To ensure that oscillations excited in the other modes decay, the system requires a small amount of damping in parallel to the springs [4].

\section{EXPERIMENTS}

In this section we experimentally validate the ability of the control to stabilize limit cycles, even when contacts with the environment occur. Therefore, we apply the control (18) and (19)-(22) to the first four joints of the DLR Hand Arm System [3] and choose the initial configuration $\boldsymbol{\theta}_{0}$,

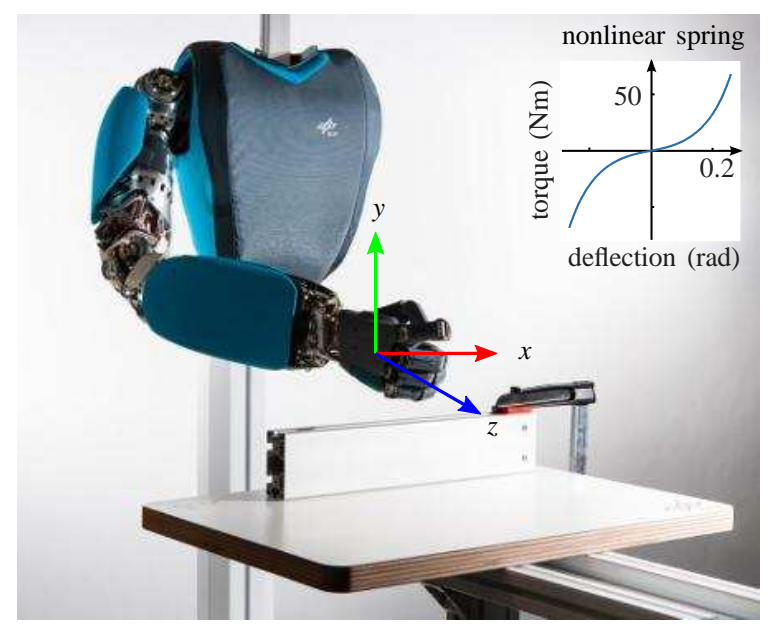

Fig. 3. Hitting experiment with the DLR Hand Arm System. The plot in the upper right corner depicts the characteristic of springs in the joints for the adjustment used in the experiments.

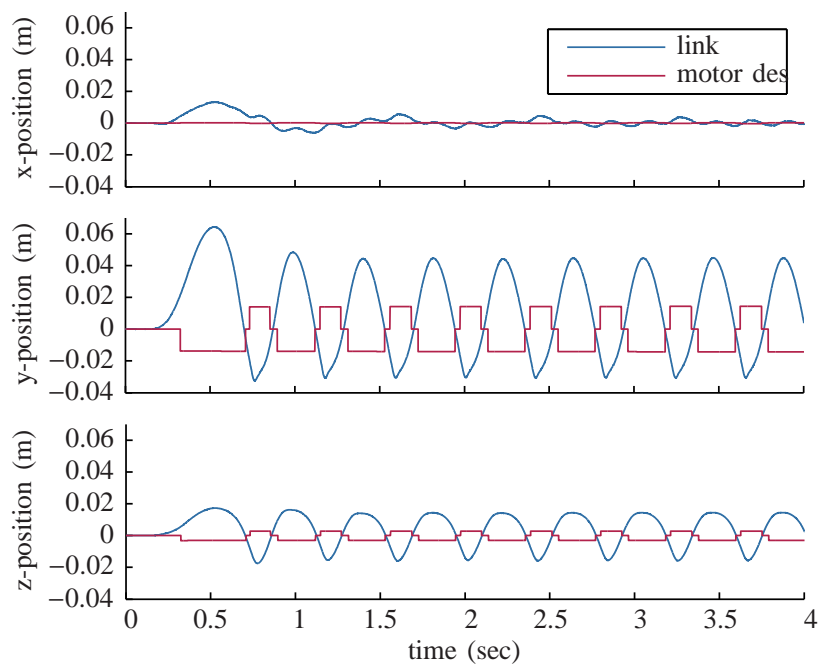

Fig. 4. Results of the hitting experiment. The plots depict the motion of the end-effector and the time evolution of the desired motor positions in terms of end-effector positions. Both representations refer to the coordinate system fixed to the ground, displayed in Fig. 3

the controller parameters, PD controller gains $\boldsymbol{K}_{P}$ and $\boldsymbol{K}_{D}$, threshold $\epsilon_{\tau_{z}}$, and switching amplitude $\hat{\theta}_{z}$, such that the endeffector gets in contact with the environment. To initially start the oscillations, the end-effector is manually deflected and released. Figure 3 shows the test setup including the robotic arm in the initial configuration. Additionally, a video showing the experiment, is attached.

In Fig. 4 it can be seen that after the initial disturbance the motion in the $x$ - and $y$-directions approaches the periodic steady state within one oscillation cycle. Thereby, in the $y$ direction, the amplitude of the positive deflection is larger than the amplitude of the negative deflection. Additionally, the trajectory of the negative deflection is sharper than in the positive direction. This is due to the end-effector hitting the environment. Moreover, Fig. 5 depicts the phase plot of the corresponding end-effector motion in the steady state. Therein, it can be seen that even in the presence of 


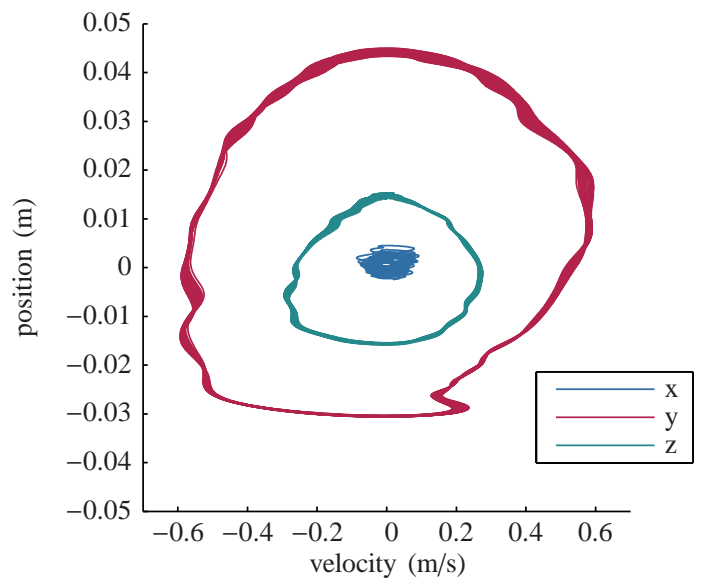

Fig. 5. Phase plot of the end-effector motion corresponding to Fig. 4 Only the steady state part of the trajectory is depicted. The position signal is lowpass filtered with a cut-off frequency of $50 \mathrm{~Hz}$. The velocity is estimated by numerical differentiation of the filtered position signal.

periodically occurring contacts, the controller stabilizes a cyclic motion within a small error band.

From the experiment with the robotic arm we conclude that the control approach might be applicable for systems with compliantly actuated legs. Hereby, hopping, jumping, walking and running constitute periodic motions of oscillatory systems that are dominated by contact sequences. The question of how to apply the control to such a type of systems, will be tackled next.

\section{Simulations}

In the following, we validate the approach with multilegged, compliantly actuated robotic systems in simulation. The biological counterparts of these systems are able to perform periodic tasks, such as jumping, hopping, walking and running. Thereby, the challenge is to handle multiple contacts and under-actuation. However, the control law described in Section $\Pi$ requires only the states of the actuated joints. No knowledge of the rigid body model, of the degree of underactuation, or of the contact state are needed. Therefore, the controller is applicable to excite periodic motions for tasks, where the degree of under-actuation changes (zero, one, or two legs are in contact) and possibly occurring sequences of contact states are not known in advance.

\section{A. Contact modeling}

We consider visco-elastic point contacts in the direction normal to the ground and viscous Coulomb friction tangential to the ground. The normal force is emulated by

$$
F_{N}=\left\{\begin{array}{ll}
-\min \left(k_{N} \Delta p_{N}+d_{N} \Delta v_{N}, 0\right) & \text { if } \Delta p_{N}<0 \\
0 & \text { otherwise }
\end{array},\right.
$$

where $\Delta p_{N}$ and $\Delta v_{N}$ are the normal components of the relative distance, respectively relative velocity, in between the foot (penetrating ground) and the initial contact point with respect to the contact surface. To approximate a rigid contact, $k_{N}$ is a large coefficient which determines the contact stiffness and $d_{N}$ is a viscous damping factor. The tangent force is emulated by the viscous Coulomb friction model

$$
F_{T}=\left\{\begin{array}{ll}
-\operatorname{sign}\left(v_{T}\right)\left|\mu F_{N}\right| & \text { if }\left|F_{T_{\mathrm{st}}}\right|>\left|\mu F_{N}\right| \\
F_{T_{\mathrm{st}}} & \text { otherwise }
\end{array} .\right.
$$

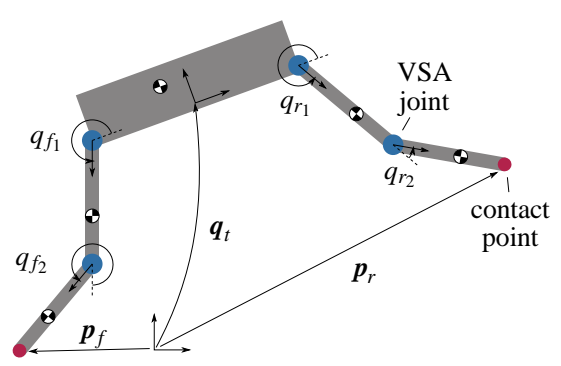

Fig. 6. Technical sketch of the planar system with two legs. The indices $f$ and $r$ denote the front and rear leg, respectively.

Herein, $F_{T_{\mathrm{st}}}$ is the stiction force, $v_{T}$ is the tangential component of the absolute velocity of the considered body-fixed point, and $\mu>0$ is the Coulomb friction coefficient. The stiction force is approximated by

$$
F_{T_{\mathrm{st}}}=-k_{T} \Delta p_{T}-d_{T} v_{T},
$$

where $\Delta p_{T}=p_{T}-p_{T_{0}}$ is the difference of the actual and initial tangent position component, $k_{T}$ is a large stiffness coefficient, and $d_{T}$ is a viscous damping factor. Finally, we consider the contact dynamics in (7) as

$$
\boldsymbol{\tau}_{\text {ext }}=\boldsymbol{\tau}_{\text {cont. }}=\sum_{i=1}^{N_{\text {cont. }}}\left(\frac{\partial \boldsymbol{p}_{i}(\boldsymbol{q})}{\partial \boldsymbol{q}}\right)^{T}\left[\begin{array}{l}
F_{T_{i}} \\
F_{N_{i}}
\end{array}\right],
$$

where $N_{\text {cont. }}$ denotes the number of body-fixed contact points and $\boldsymbol{p}_{i}(\boldsymbol{q})$ is the absolute position of the $i$-th body-fixed contact point.

\section{B. A planar system with two legs}

The system consists of a trunk and two double pendulumlike legs. As schematically sketched in Fig. 6, the trunk (main body) has two translational and one rotational degrees of freedom. Thereby, the generalized coordinates $\boldsymbol{q}_{t} \in \mathbb{R}^{3}$ represent the pose of the trunk. The upper legs are hinged to the trunk and the lower legs are hinged to the upper legs. Thereby, $\boldsymbol{q}_{\text {joint }}=\left(q_{r_{1}}, q_{r_{2}}, q_{f_{1}}, q_{f_{2}}\right)^{T}$ represents the configuration of the rear and front legs, respectively. The distance between the suspension points of the legs is denoted $l_{t}$ and the link length of the upper and lower legs $l_{j}$, where $j=$ $r_{1}, r_{2}, f_{1}, f_{2}$, corresponds to the indices of the joint positions $\boldsymbol{q}_{\text {joint }}$ (see, Fig. 6). The mass distributions of the trunk and links are modeled as point masses $m_{t}$ respectively $m_{j}$, where the positions of the point masses w.r.t. to the body-fixed frame $c_{t_{x}}$ and $c_{t_{v}}$ respectively $c_{j}$ are introduced as parameters. A contact point (cf. Section IV-A is attached to each lower leg. The hip and knee joints are equipped with serial elastic actuators. To analyze the influence of a nonlinear spring characteristic, we consider the elastic potential

$$
U_{\psi}\left(\boldsymbol{q}_{\text {joint }}, \boldsymbol{\theta}\right)=\sum_{j}\left(\frac{1}{2} k_{1, j}\left(q_{j}-\theta_{j}\right)^{2}+\frac{1}{4} k_{2, j}\left(q_{j}-\theta_{j}\right)^{4}\right)
$$

with $k_{1, j}>0$ and $k_{2, j} \geq 0$. Notice that $k_{2, j}=0$ corresponds to a linear and $k_{2, j}>0$ to a cubic deflection-force relation. Additionally, weak joint damping of the form

$$
\boldsymbol{D} \dot{\boldsymbol{x}}=\left[\begin{array}{ccc}
\boldsymbol{D}_{\text {joint }} & -\boldsymbol{D}_{\text {joint }} & \mathbf{0} \\
-\boldsymbol{D}_{\text {joint }} & \boldsymbol{D}_{\text {joint }} & \mathbf{0} \\
\mathbf{0} & \mathbf{0} & \mathbf{0}
\end{array}\right]\left[\begin{array}{c}
\dot{\boldsymbol{\theta}} \\
\dot{\boldsymbol{q}}_{\text {joint }} \\
\dot{\boldsymbol{q}}_{t}
\end{array}\right],
$$


where $\boldsymbol{D}_{\text {joint }} \in \mathbb{R}^{4 \times 4}$ is diagonal and positive, acts in parallel to the springs (cf. (7) in Section III). Finally, we consider the states of the actuated joints as measurable and compute the control input $\boldsymbol{u} \in \mathbb{R}^{4}$ in (7) using (18) and (19)-(22) described in Section (II).

C. Comparison of a sinusoidal excitation and the modal bang-bang control for a jumping motion

Initially, we consider a jumping motion for the system described above and compare a sinusoidal excitation and the modal bang-bang control. The system parameters and the initial configuration are given in Table \. First, we test a sinusoidal excitation as desired motor position $\boldsymbol{\theta}_{d}(t)=\boldsymbol{q}_{\text {joint }}\left(t_{0}\right)+$ $\hat{\boldsymbol{a}} \sin (8 \pi t)$, with amplitudes $\hat{\boldsymbol{a}}=(0.2,-0.4,-0.2,0.4)^{T}$. Second, we compare it to the modal bang-bang control, with controller settings $\epsilon_{\tau_{z}}=4, \hat{\theta}_{z}=\|\hat{\boldsymbol{a}}\|$ and initial conditions for the adaptive part $\boldsymbol{w}_{1}\left(t_{0}\right)=\hat{\boldsymbol{a}} /\|\hat{\boldsymbol{a}}\|$. For both cases (feedforward and feedback control) the simulation starts with an initial trunk height $q_{t_{2}}\left(t_{0}\right)=0.9 \mathrm{~m}$ and zero velocities.

As can be seen from Fig. 7 (and the video attachment), we obtain a jumping motion for both types of excitations. For the sinusoidal excitation, the trajectory of the vertical trunk position does not converge to a periodic motion during the considered simulation time. Using the modal bang-bang control, the trajectory converges to a limit cycle within a small error band. It should be mentioned that in the case of the sinusoidal excitation, the frequency has been chosen arbitrarily. Therefore, the frequency does not match the intrinsic frequency of the jumping task. In further simulations (not described here) it turned out that manually tuning the frequency and phase of the sinusoidal excitation improves the matching of the frequency to the task. In contrast the modal bang-bang control adapts itself to the intrinsic frequency of the system/task.

TABLE I

PARAMETERS OF THE JUMPING SYSTEM

\begin{tabular}{c||c|c|c|c|c} 
index $i$ & trunk $t$ & rear $r_{1}$ & rear $r_{2}$ & front $f_{1}$ & front $f_{2}$ \\
\hline \hline$m_{i}(\mathrm{~kg})$ & 2.0 & 0.1 & 0.1 & 0.1 & 0.1 \\
\hline$l_{i}(\mathrm{~m})$ & 0.9 & 0.25 & 0.2 & 0.25 & 0.2 \\
\hline$c_{i}(\mathrm{~m})$ & 0,0 & 0.125 & 0.1 & 0.125 & 0.1 \\
\hline$k_{1, i}(\mathrm{Nm} / \mathrm{rad})$ & & 70 & 70 & 70 & 70 \\
\hline$k_{2, i}(\mathrm{Nm} / \mathrm{rad})$ & & 0 & 0 & 0 & 0 \\
\hline$d_{i}(\mathrm{Nms} / \mathrm{rad})$ & & 1 & 1 & 1 & 1 \\
\hline$q_{i}\left(t_{0}\right)(\mathrm{deg})$ & & -120 & 70 & -60 & -70
\end{tabular}

\section{From jumping to forward hopping by changing the mass} distribution and the stiffness characteristics

In order to excite a forward hopping mode, we slightly change the parameters considered for the jumping system. For a first test, we keep the parameters and the initial configuration given in Table $\Pi$ and shift only the point mass of the trunk such that $c_{t_{x}}=-0.2 \mathrm{~m}$. This already leads to a forward hopping motion, which decays as the energy input due to the bang-bang control is smaller than the dissipated energy. To increase the energy input due to the bang-bang control, we change the spring characteristic such that $k_{2, j}=10 k_{1, j}$. Notice that introducing a progressive spring characteristic increases the input energy for the unmodified switching amplitude $\hat{\theta}_{z}$.

The resulting forward hopping motion is visualized in Fig. 8 (and the video attachment). As can be seen in Fig. 9
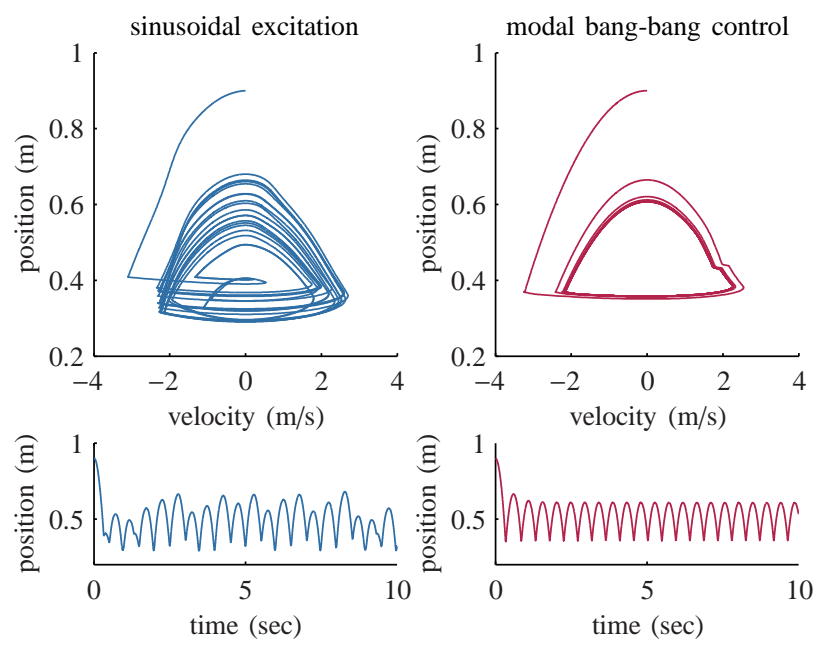

Fig. 7. Comparison of a feedforward sinusoidal excitation and the modal bang-bang control for a hopping motion.

the trunk performs a pitch oscillation, while moving forward from right to left. After the initial transient, the trajectory of joint positions form closed orbits in the phase space within an error band (see, Fig. 10). Moreover, the contact forces are depicted in Fig. 11. It can be seen that a flight phase appears periodically. This phase is longer than the phase when one or both feet are in contact with the ground.

Due to a slight change of the mechanical parameters, mass distribution and spring characteristic, the intrinsic system properties change and evolve from jumping towards a forward hopping mode. Thereby, each dominant mode can be excited by the modal bang-bang control.

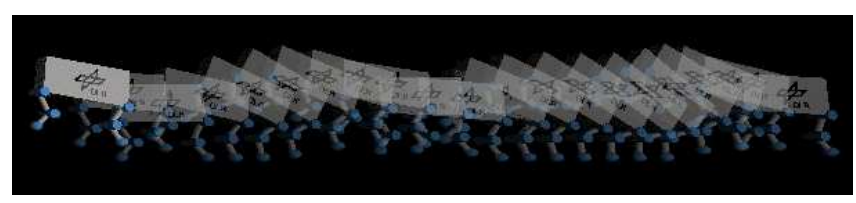

Fig. 8. Image sequence of the forward hopping motion. The picture shows approximately two motion cycles. Thereby, the motion evolves from right to left. For reason of clarity, each image is horizontally shifted by a constant offset.

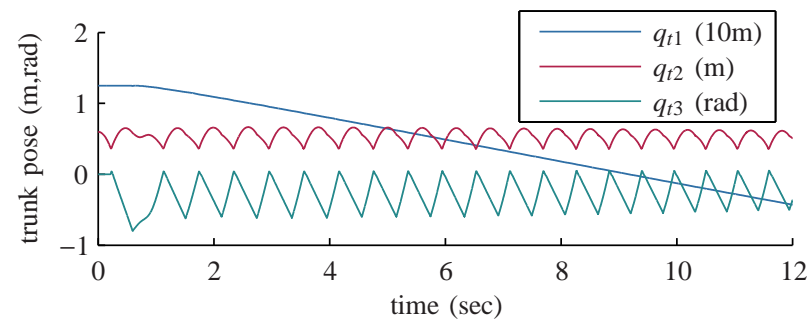

Fig. 9. Time evolution of the trunk pose for the forward hopping motion The coordinates $q_{t_{1}}$ and $q_{t_{2}}$ represent the horizontal respectively vertical position and $q_{t_{3}}$ the orientation of the trunk.

\section{E. A configuration predestined to excite a running mode}

Due to the configuration dependent inertia matrix, the initial configuration might influence which oscillation mode 
Fig. 12. Image sequence of the running motion in a "crab" configuration. The picture shows approximately two motion cycles. Thereby, the motion evolves from right to left. For reason of clarity, each image is horizontally shifted by a constant offset.

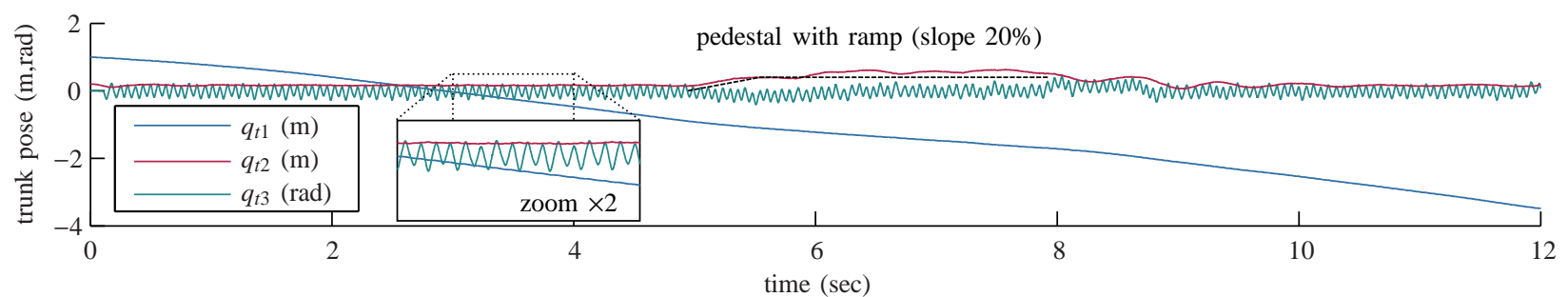

Fig. 13. Time evolution of the trunk pose for the running motion. The coordinates $q_{t_{1}}$ and $q_{t_{2}}$ represent the horizontal respectively vertical position and $q_{t_{3}}$ the orientation of the trunk.

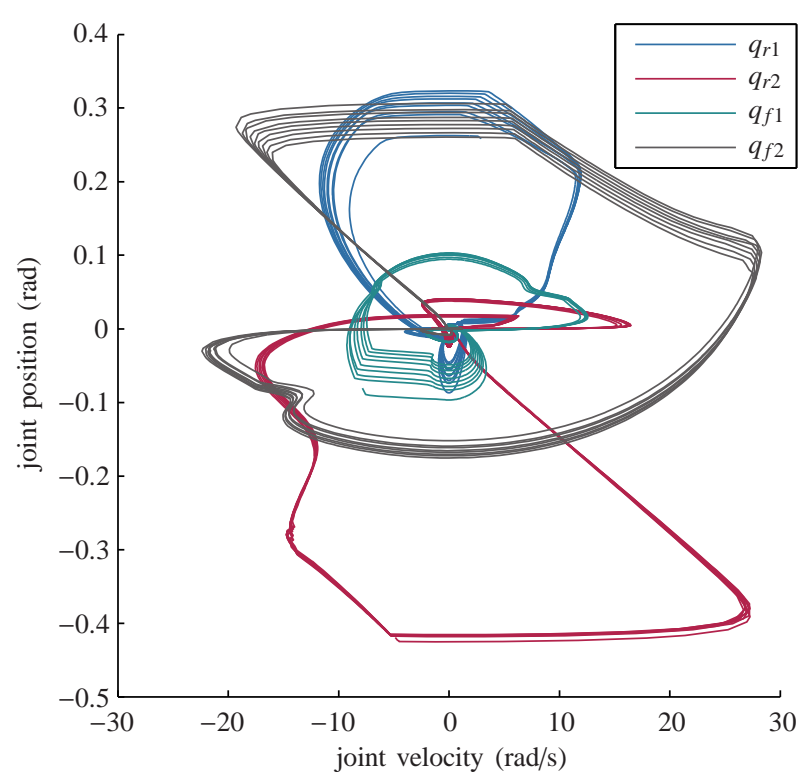

Fig. 10. Phase plot of joint motion in the steady-state phase of the forward hopping motion.

becomes dominant. In the following, we change the initial configuration such that the controlled system tends to converge to a running mode. The system from Section IV-C and IV-D corresponds to an idealized "quadrupedal animal" configuration, while the system considered in this section represents rather a "crab" configuration. Therefore, we consider the parameters and the initial configuration given in Table III The bang-bang threshold is adjusted to $\epsilon_{\tau}=2$ such that the controller switches due to gravity. Moreover, the switching amplitude $\hat{\theta}_{z}=0.8$ and the initial condition of the adaptive part $\boldsymbol{w}_{1}\left(t_{0}\right)=(1,0,0,0)^{T}$. In initial tests, it turned out that the adaptive part always converges to the same values $\hat{\boldsymbol{w}}_{1}$. Therefore, in further simulations, we set $\boldsymbol{w}_{1}\left(t_{0}\right)=\hat{\boldsymbol{w}}_{1}$.

The resulting running motion is visualized in Fig. 12 (and the attached video). To test the robustness against a disturbance, we introduce an obstacle in form of a pedestal with a ramp at one side. As can be seen from the trunk

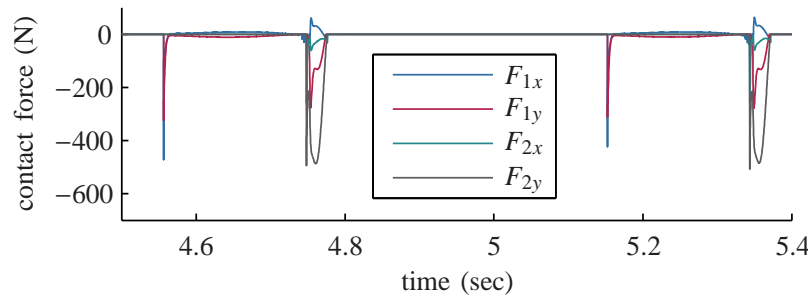

Fig. 11. Contact forces acting in the steady-state phase of the forward hopping motion. The components $F_{r_{x}}$ and $F_{r_{y}}$ correspond to the horizontally respectively vertically acting forces of the rear foot (cf. Fig. 6). The components $F_{f_{x}}$ and $F_{f_{y}}$ correspond analogously to the front foot. Due to under-sampling effects, the signal is not periodical.

motion depicted in Fig. 13, the controlled system is able to robustly handle this disturbance and converges back to the initial running motion. Furthermore, the corresponding contact forces are depicted in Fig. 14 It can be seen that long flying phases alternate with short contact phases.

TABLE II

PARAMETERS OF THE RUNNING SYSTEM

\begin{tabular}{c||c|c|c|c|c} 
index $i$ & trunk $t$ & rear $r_{1}$ & rear $r_{2}$ & front $f_{1}$ & front $f_{2}$ \\
\hline \hline$m_{i}(\mathrm{~kg})$ & 2.0 & 0.1 & 0.1 & 0.1 & 0.1 \\
\hline$l_{i}(\mathrm{~m})$ & 1.0 & 0.5 & 0.5 & 0.5 & 0.5 \\
\hline$c_{i}(\mathrm{~m})$ & $0,0.25$ & 0.25 & 0.25 & 0.25 & 0.25 \\
\hline$k_{1, i}(\mathrm{Nm} / \mathrm{rad})$ & & 70 & 84 & 70 & 84 \\
\hline$k_{2, i}(\mathrm{Nm} / \mathrm{rad})$ & & 0 & 0 & 0 & 0 \\
\hline$d_{i}(\mathrm{Nms} / \mathrm{rad})$ & & 1 & 1 & 1 & 1 \\
\hline$q_{i}\left(t_{0}\right)(\mathrm{deg})$ & & 30 & -90 & 150 & 90
\end{tabular}

\section{F. Comments on the contact forces}

As can be seen in Fig. 11 and 14 the peaks of the contact forces occurring during the forward jumping respectively running motion are rather high with respect to the total mass of the system. The high peak forces result due to a combined effect: the switching in the bang-bang control (cf. (20)-(22) in Section (II) and the relatively high damping in the joints (compared to the inertia and stiffness). From these observations, one can deduce that the joints of the robotic 


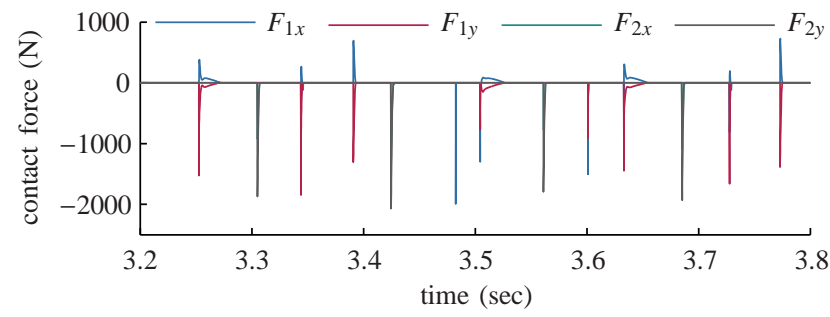

Fig. 14. Contact forces acting during the running motion. The components $F_{r_{x}}$ and $F_{r_{y}}$ correspond to the horizontally respectively vertically acting forces of the rear foot (cf. Fig. 6. The components $F_{f_{x}}$ and $F_{f_{y}}$ correspond analogously to the front foot.

system have to be designed such that the damping is as low as possible. However, there is a lower bound for the damping in parallel to the springs, which ensures the decay of oscillations excited in the undesired modes [4].

\section{G. Summary of the simulation results}

The preceding simulation study demonstrates that the proposed control method appropriately excites periodic motions in compliantly actuated, legged robotic systems. In particular, the feedback controller is able to adapt to the intrinsic frequency of the system and task. This is a clear advantage compared to a feedforward excitation. Furthermore, changing the mechanical parameters or the initial configuration of the systems leads to a different motion pattern. This indicates that the controller excites dominantly appearing oscillation modes, which correspond to intrinsic properties of the uncontrolled mechanical systems. Therefore, the modal bang-bang control might be used to derive rough guidelines for choosing a design for a mechanical system, which is appropriate for performing a specific motion pattern. Finally, it should be remarked that to reach a desired mode of motion, the most challenging issue is to find a proper initial excitation, mainly determined by the initial condition of the adaptive part $\boldsymbol{w}_{1}\left(t_{0}\right)$ (cf. (18) in Section II). A directed method to find an appropriate initial condition $\boldsymbol{w}_{1}\left(t_{0}\right)$ will be treated in a future work.

\section{Conclusion}

A modal bang-bang controller is presented that excites periodic motions in compliantly actuated robotic systems, in the presence of robot-environment contacts. The approach is experimentally tested on the DLR Hand Arm System and has shown to robustly perform a hitting motion. The controller requires only measurements of the states of the actuated joints and no model knowledge. Therefore, as shown in simulation, the approach can be straightforwardly applied to legged robotic systems with compliantly actuated joints such that the controlled system is able to execute periodic tasks such as jumping, forward hopping, and running.

For a planar system with two legs, a simulation study is performed. Thereby, the modal bang-bang controller is compared to a feedforward sinusoidal excitation and the influence of the mechanical parameters as well as the kinematic leg configuration is analyzed. Simulations of a jumping motion show that the modal bang-bang controller adapts to the jumping mode and synchronizes the task-intrinsic frequency. Furthermore, it is shown that by changing the mechanical parameters of the system and/or the equilibrium configuration of the legs, the system evolves from a jumping to a forward hopping and to a running mode. This indicates that the controller excites intrinsic mechanical modes of the system and task. Therefore, the approach is a useful tool to find some design guidelines for such challenging systems.

\section{ACKNOWLEDGMENT}

This work has been partially funded by the European Commission's Seventh Framework Programme as part of the project SAPHARI (Grant no. 287513).

\section{REFERENCES}

[1] S. Wolf and G. Hirzinger, "A new variable stiffness design: Matching requirements of the next robot generation," in IEEE Int. Conf. on Robotics and Automation, 2008.

[2] A. Albu-Schäffer, O. Eiberger, M. Fuchs, M. Grebenstein, S. Haddadin, C. Ott, A. Stemmer, T. Wimböck, S. Wolf, C. Borst, and G. Hirzinger, "Anthropomorphic soft robotics - from torque control to variable intrinsic compliance," in Robotics Research, ser. Springer Tracts in Advanced Robotics, C. Pradalier, R. Siegwart, and G. Hirzinger, Eds. Springer, 2011, vol. 70, pp. 185-207.

[3] M. Grebenstein, A. Albu-Schäffer, T. Bahls, M. Chalon, O. Eiberger, W. Friedl, R. Gruber, S. Haddadin, U. Hagn, R. Haslinger, H. Höppner, S. Jörg, M. Nickl, A. Nothhelfer, F. Petit, J. Reill, N. Seitz, T. Wimböck, S. Wolf, T. Wüsthoff, and G. Hirzinger, "The DLR Hand Arm System," in Proc. IEEE Int. Conf. on Robotics and Automation, 2011.

[4] D. Lakatos, F. Petit, and A. Albu-Schäffer, "Nonlinear oscillations for cyclic movements in variable impedance actuated robotic arms," in IEEE Int. Conf. on Robotics and Automation 2013, 2013.

[5] R. Full and D. Koditschek, "Templates and anchors: neuromechanical hypotheses of legged locomotion on land," J. Exp. Biol., vol. 202, no. 15, pp. 3325-3332, 1999.

[6] J. Buchli and A. Ijspeert, "Self-organized adaptive legged locomotion in a compliant quadruped robot," Autonomous Robots, vol. 25, no. 4 , pp. 331-347, 2008 .

[7] A. Ijspeert, "A connectionist central pattern generator for the aquatic and terrestrial gaits of a simulated salamander," Biol. Cybern., vol. 84, pp. 331-348, 2001.

[8] A. Crespi and A. J. Ijspeert, "Amphibot ii: An amphibious snake robot that crawls and swims using a central pattern generator," in Int. Conf. on Climbing and Walking Robots, 2006, pp. 19-27.

[9] A. J. Ijspeert and A. Crespi, "Online trajectory generation in an amphibious snake robot using a lamprey-like central pattern generator model," in IEEE Int. Conf. on Robotics and Automation, 2007, pp. 262-268.

[10] P. Kokotovic, H. Khalil, and J. O’Reilly, Singular Perturbation Methods in Control: Analysis and Design. Academic Press, London, 1986.

[11] A. Albu-Schäffer, C. Ott, and F. Petit, "Constructive energie shaping control for a class of euler-lagrange systems," in 10th Int. IFAC Symposium on Robot Control, 2012.

[12] M. Kirby and R. Miranda, "Nonlinear reduction of high-dimensional dynamical systems via neural networks," Physical Review Letters, vol. 72, pp. 1822-1825, Mar 1994.

[13] T. Frankel, The Geometry of Physics: An Introduction, 2nd ed. Cambridge University Press, 2003.

[14] R. Horn and C. Johnson, Matrix Analysis. Cambridge University Press, 1985.

[15] E. Oja, "Principal components, minor components, and linear neural networks," Neural Networks, vol. 5, pp. 927-935, 1992.

[16] - Subspace methods of pattern recognition. Letchworth England: Research Studies Press and John Wiley and Sons, 1983.

[17] T. Sanger, "Optimal unsupervised learning in a single-layer linear feedforward neural network," Neural Networks, vol. 2, pp. 459-473, 1989.

[18] B. Feeny and R. Kappagantu, "On the physical interpretation of proper orthogonal modes in vibrations," Journal of Sound and Vibration, vol. 211, pp. 607-616, 1998.

[19] S. Shaw and C. Pierre, "Normal modes for non-linear vibratory systems," Journal of Sound and Vibration, vol. 164, no. 1, pp. 85124, 1993. 